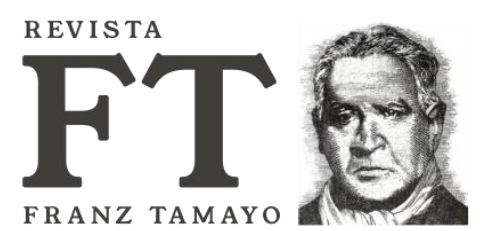

\title{
La supervisión educativa. Incidencia en las políticas públicas
}

\author{
Educational supervision. Impact on public policies
}

Recibido junio 2019 Arbitrado julio 2019

Publicado septiembre 2019
Leida Ayary Flores

ayari970@hotmail.com

Código ORCID: 0000-0001-7693-7842

Gobierno del Distrito Capital- Secretaría para la Educación, Venezuela

\section{Resumen}

La presente investigación tuvo como objetivo teorizar acerca del sistema de apoyo gerencial para la sustentabilidad de la supervisión educativa y su incidencia en las políticas públicas, con el fin de unificar criterios en cuanto al desempeño de la supervisión educativa por parte de quienes ejercen la función supervisora en las unidades educativas y los circuitos educativos. La ruta metodológica respondió a una investigación cualitativa, apoyada en el método fenomenológico que comprendió el registro, análisis e interpretación de la naturaleza actual de la realidad vivida y experimentada por seis informantes claves: un docente de aula, dos coordinadores y tres supervisores de nivel central, a quienes se les aplicó una entrevista focalizada. El análisis de la información permitió concluir que los supervisores desconocen los procesos gerenciales de supervisión y dirección que rigen para el establecimiento de las políticas públicas y que existe una ausencia de un sistema de supervisión.

\footnotetext{
Abstract

This research aimed to theorize about the management support system for the sustainability of educational supervision and its impact on public policies, in order to unify criteria regarding the performance of educational supervision by those who exercise the supervisory function in educational units and educational circuits. The methodological route responded to a qualitative investigation, supported by the phenomenological method that included the registration, analysis and interpretation of the current nature of the reality lived and experienced by six key informants: a classroom teacher, two coordinators and three central level supervisors. , to whom a focused interview was applied. The analysis of the information allowed us to conclude that the supervisors are unaware of the managerial processes of supervision and direction that govern the establishment of public policies and that there is an absence of a supervisory system.
}

Palabras clave:

Supervisión educativa; sistema de apoyo gerencial; políticas públicas; sustentabilidad; resignificación

\author{
Keywords: \\ Educational \\ supervision; \\ management \\ support system; \\ public policies; \\ sustainability; \\ resignification
}




\section{INTRODUCCIÓN}

H $\mathrm{n}$ el proceso de transformación y cambios del sistema educativo venezolano, se ha observado incremento de la matrícula escolar, a partir del surgimiento de las escuelas bolivarianas como políticas públicas educativas. Sin embargo, para el año 2019, se evidencia deserción escolar por migración de los estudiantes, debido a la crisis económica que presenta el país. No obstante, para lograr estos cambios que ameritan atender la demanda científica y tecnológica de los últimos tiempos, se requiere de profesionales capacitados que participen en los procesos educativos atendiendo a las nuevas políticas y esquemas que necesita el país (Maldonado, 2008).

Estos cambios incluyen la supervisión, por considerarse la base fundamental de la educación, ya que su función está fundamentada en los principios que rigen la educación, como lo establece la Constitución de la República Bolivariana de Venezuela (CRBV, 1999), basado en el trabajo en equipo al estimular y facilitar los medios que conducen, a través de su acción al crecimiento profesional del docente, a la planificación científica ajustada a las necesidades y al cumplimiento de las actividades, en los procesos educativos para la creación de una sociedad más justa, más humana, donde la escuela sea un espacio abierto para la formación integral, la creación, la creatividad y las innovaciones en las prácticas pedagógicas que contribuyan al desarrollo personal del individuo.

En este sentido, la supervisión educativa es un proceso dinámico y sistemático que tiene como objeto garantizar el cumplimiento de los fines y propósitos del Estado como política educativa; tiene como misión orientar, asesorar, controlar, coordinar y evaluar mediante un ejercicio participativo y cooperativo, y de allí los cambios y las transformaciones que se dan para mejorar la educación.

Por lo antes expuesto, la supervisión educativa requiere de atención y debe ser orientada para ayudar al docente a resolver los problemas concretos, y no centrada en una práctica profesional de vigilancia y ubicación de fallas para aplicar correctivos superficiales, sino que evalúe y mejore los factores que afectan a la organización y al proceso enseñanza y aprendizaje.

En este orden de ideas, la problemática de la supervisión educativa en Venezuela, según las bibliografías consultadas Bonilla 
(2014), Mogollón (2006), Néreci (1975), Ruiz (2005) entre otros documentos, se manifiesta en el hecho de que no cumple su función de manera eficiente, se presenta una supervisión deficiente y punitiva, de ausencia del talento humano, de carencia de formación y descontextualizada, de desconocimiento de los procesos administrativos y pedagógicos, de dirección y supervisión, de normativa legal, de debilidad en asesorar a los docentes antes, durante y después del ejercicio, de las herramientas necesarias que le sirvan de apoyo para la solución de sus problemas.

Por otra parte, el sistema de supervisión vigente ha mostrado debilidad al dirigir y controlar la ejecución de estos procesos, por lo que se requiere la necesidad de la reestructuración del sistema de supervisión, prestándole atención técnica y profesional, para que puedan promover el rendimiento de la organización educativa; puesto que cuando el supervisor utiliza su capacidad cognoscitiva y ejercer su rol de líder propicia el cambio de las personas y hace que estas se adapten a nuevos trabajos, enfoques, estrategias, que sean flexibles, capaces de que se trabaje en grupos heterogéneos, pero motivados, cargados de energía, dispuestos al logro de las metas (Ruiz, 2005).

En efecto, estas debilidades son contrario a los objetivos de la supervisión educativa que tiene la tarea de garantizar el cumplimiento de los fines y propósitos del Estado venezolano, ya que la supervisión educativa debe ubicarse a la altura de las nuevas exigencias en todos los niveles y modalidades del sistema educativo, para orientar, encauzar, evaluar y garantizar la eficacia del hecho pedagógico de la organización educativa.

Por ello, para Vásquez (2016):

... la educación debe contar con supervisores debidamente capacitados con herramientas pertinentes para garantizar el éxito del hecho educativo y del cumplimiento de las políticas públicas tanto, en lo administrativo, como pedagógico, a fin de que se apliquen efectivamente los procesos inherentes a la supervisión, tales como: un proceso único, integral, holístico, social, humanista, sistemático, metodológico, planificador, que realice control, evaluación, coordinación, orientación, asesoramiento, que sea líder, que motive, que propicie la comunicación y la toma de decisiones. (s.n.) 
En este contexto, pueden existir las mejores propuestas, pero el éxito depende de que se ejecute, su control, el asesoramiento y la evaluación, de lo contrario se estaría siempre conduciendo al fracaso (Ocando, 2017).

$\mathrm{El}$ autor anteriormente citado refiere que vale la pena resaltar que la verdadera razón del fracaso de las políticas educativas radica en no haber logrado concienciar que es el docente quien, a través de una supervisión educativa ajustada a los cambios sociales, responda a principios democráticos y ejecute la transformación que se requiere.

En consecuencia, los cambios en las políticas públicas y el rol de la supervisión educativa para pasar de lo administrativo del sistema educativo a conducir los procesos pedagógicos de calidad en correspondencia con las políticas públicas son subjetivos. Por todas las razones antes expuestas, se presenta esta investigación, la cual se planteó como objetivo general:

Teorizar acerca de los sistemas de apoyo gerencial para la sustentabilidad de la supervisión educativa y su incidencia en las políticas públicas.

Y como objetivos específicos:

- Conocer el sustento teórico que fundamenta el sistema de apoyo gerencial para la sustentabilidad de la supervisión educativa.

- Indagar sobre las políticas públicas que dan sustento a la supervisión educativa.

- Develar las funciones de los supervisores educativos para el alcance de las políticas públicas y las prácticas pedagógicas.

\section{MÉTODO}

$\mathrm{E}$ l presente estudio se enmarcó en una investigación cualitativa, descriptiva, inductiva, fenomenológica, holística, ecológica, estructural, sistemática, de diseño flexible, que permitió hacer descripciones detallas de las situaciones, eventos y comportamientos observados en el estudio.

En cuanto al paradigma cualitativo, Martínez (2004) sostiene que, ante la insatisfacción de los métodos de investigación y los criterios de validación del paradigma tradicional, surge este como un enfoque alterno que denomina investigación estructural para dar 
respuesta al estudio de las realidades sociales. En él se incluyen la hermenéutica, la fenomenología, la etnografía, investigación acción, la historia de vida, entre otros.

La orientación epistemológica que se asumió en esta investigación, se fundamenta en la fenomenología social de Schütz (1974), quien desarrolló una aproximación fenomenológica a lo social, a lo cotidiano, a la experiencia de los sujetos, una fenomenología del mundo social sobre la base de la experiencia diaria, una relacionada con la vivencia interna de las personas y que trata de reconstruir el mundo de acuerdo con la experiencia que tiene el hombre.

El método fenomenológico consta de cuatro etapas y pasos, a saber (Martínez, 2004):

a) Etapa previa: se clarifican los presupuestos.

b) Etapa descriptiva: se pretendió lograr que la descripción del fenómeno sea lo más completa y desprejuiciada posible y que reflejara la realidad vivida por el sujeto que se investiga, su mundo, situaciones, características. Aquí se hizo la elección de la técnica apropiada, como por ejemplo la observación, la entrevista; realización de esas técnicas y la elaboración de la descripción protocolar, donde se reflejó la realidad tal y como se presentó, que sea lo más completa posible, que no se omita nada, que no contenga elementos proyectados y que revele al fenómeno tal y como se está viviendo, sin colocarlo en otro ambiente.

c) Etapa estructural: descripción de los protocolos, constó de seis pasos:

1. Lectura general de la descripción de cada protocolo y la descripción de los protocolos con la actitud de revivir la realidad en una situación concreta y luego reflexionara acerca de la situación estudiada.

2. Delimitación de las unidades temáticas naturales. Esto consiste en pensar, meditando, sobre el posible significado que pudiera tener una parte en un todo.

3. Determinación del tema central que domina cada unidad temática. 
4. Expresión del tema central en un lenguaje científico.

5. Integración de todos los temas centrales en una estructura particular descriptiva.

6. Descubrir la estructura del fenómeno investigado.

d) Etapa de discusión de hallazgos: relacionar las proposiciones teóricas y antecedentes investigativos con lo expresado por los sujetos informantes de la investigación.

La naturaleza de la investigación fue interpretativa por cuanto tal y como lo expresa Husserl (1962), tendrá como propósito "hacer evidente la experiencia humana a través de la reflexión y así descubrir las formas genuinas de los propios pensamientos" (p. 229). Según este autor, desde esta perspectiva se hace referencia a formas concretas de percibir y abordar la realidad, lo cual lleva a compartir posiciones desde lo multirreferencial cambiante y cuyas explicaciones son un producto social, los conceptos y marcos de interpretación deben construirse a partir del análisis directo del mundo social y no desde ideas preconcebidas.

El tipo de la investigación fue de campo, enmarcada en lo fenomenológico por estar sustentada en el diseño de investigación cualitativo. Comprendió el registro, análisis e interpretación de la naturaleza actual y la composición o procesos de los fenómenos. Para Rojas (2010), una investigación de campo implica que la información se obtiene directamente de la realidad donde ocurren los hechos.

Este trabajo se ubicó en este tipo y diseño de investigación por cuanto la información se obtuvo de los docentes y supervisores adscritos al nivel central del MPPE y de las coordinaciones del Gobierno del Distrito Capital, las cuales aportaron su visión gerencial de la supervisión educativa ante la propuesta de contribuir y enriquecer la praxis educativa.

Para concretar la información a recolectar, los sujetos de investigación o informantes claves fueron tres (3) supervisores zonales y distritales, dos (2) directores de instituciones educativas, y un (1) docente de aula, para un total de seis (6) sujetos (ver Gráfico 1). 


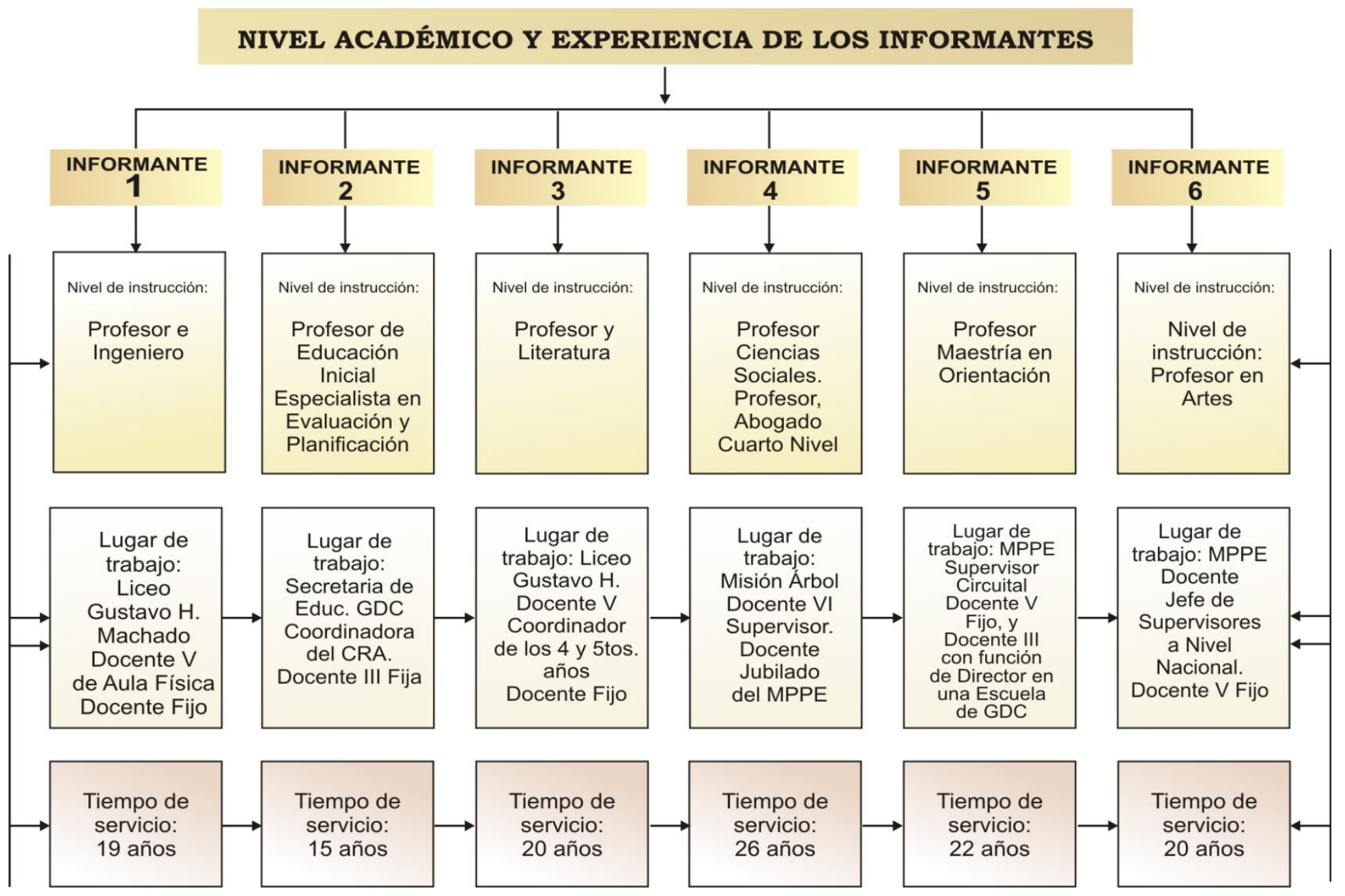

Gráfico 1. Nivel académico y experiencia de los informantes claves 
La recolección de información se realizó mediante la observación participativa y la entrevista en profundidad focalizada, de manera individual y dirigida.

La observación participativa como elemento clásico primario utilizada por los investigadores, es aquella donde el investigador se incluye en el grupo, hecho o fenómeno observado, para obtener información "desde adentro". Implica un trabajo dilatado y cuidadoso (Palella y Martins, 2004).

Por otra parte, se empleó la entrevista en profundidad focalizada para lograr una aproximación fenomenológica a la vivencia de los profesionales frente al conocimiento de la supervisión educativa en los planteles. En este caso, se pone en vigencia los planteamientos de Taylor y Bogdan (1987), quienes señalan que las entrevistas corresponden a los reiterados encuentros cara a cara que el investigador sostiene con el o los informante (s). Estos encuentros persiguen comprender las perspectivas u opiniones que los sujetos del estudio tienen respecto de sus experiencias o situaciones concretas expuestas con sus propias palabras.

El instrumento utilizado fue el guión de entrevista, el cual se conformó con aspectos claves que se desean conocer sobre el tópico a investigar.

La información que se obtuvo mediante el empleo de la entrevista, se validó a través de la triangulación la cual supone que los resultados se ajustan a la interpretación del significado de las expresiones emitidas por los entrevistados. El objetivo de la validación en una investigación cualitativa es tener la voluntad de escuchar y darle "voz a los entrevistados y representarlos tan precisamente como sea posible" (Strauss y Corbin, 2002, p. 48).

Para este estudio se utilizó la triangulación de espacio, de evaluador, metodológica, teórica y el rechequeo con los sujetos (Rojas, 2010). Para Pérez (1998), la triangulación es el proceso que consiste en "un control cruzado entre diferentes fuentes de datos, personas, instrumentos, documentos o la combinación de estos" (p. 81), lo que permitirá descubrir las semejanzas o patrones subyacentes de la praxis en la Supervisión en el contexto educativo, así como sus diferencias para obtener una comprensión profunda y clara de la realidad estudiada.

En el mismo orden de ideas se hace referencia a la triangulación teórica, la cual permitió abordar el estudio desde diversas perspectivas teóricas y facilitó al investigador desplazarse entre 
ellas, según Rojas (2010). En esta investigación, se hizo un contraste entre las proposiciones teóricas analizadas y las respuestas dadas por cada uno de los informantes. En consecuencia, para Denzin y Lincoln (2005), esto implica la categorización, la estructuración, la contrastación y la teorización, ellos son esencialmente críticos y evaluativos, es decir, que en su devenir juega continuamente con alternativas posibles para elegir la mejor categoría para una determinada información, la estructura que da explicaciones más plausibles para un conjunto de categorías y la teoría que mejor integra las diferentes estructuras en un todo coherente y lógico.

\section{RESULTADOS}

$\mathrm{E}$ ste aspecto de la investigación tiene por objetivo sistematizar los resultados del procesamiento de la entrevista focalizada realizada a un grupo de profesionales de la educación, conocedores del tópico con la intención de dar a conocer el análisis de estos y abarcar la descripción de la totalidad de las categorías.

La acción se centró en el análisis de las categorías: a) sistema de apoyo gerencial educativo; b) funciones o procesos gerenciales; c) programas, proyectos y leyes; d) ascenso por filiación política; e) normas jurídicas.

Los resultados permitieron valorar el logro de los objetivos específicos formulados:

\section{- Conocer el sustento teórico que fundamenta el sistema de apoyo gerencial para la sustentabilidad de la supervisión educativa.}

Este objetivo responde a todas las categorías: supervisión punitiva; carencia de formación del supervisor o la supervisora; carencia de las prácticas pedagógicas y administrativas; programas, proyectos y leyes; ascenso por filiación política. En este sentido, en los Cuadros 1 y 2 se observan las debilidades y las fortalezas, respectivamente. 
Cuadro 1. Debilidades del sistema de apoyo gerencial para la sustentabilidad de la supervisión educativa.

\section{DEBILIDADES}

1. Desconocimientos de teorías epistemológicas que le dan sustento a un sistema, y las diferencias ciencias que conjugan y da apoya gerencial de la supervisión educativa.

2. Desconocimientos de los procesos dirección y supervisión

3. Ausencia de supervisión, va a la escuela cuando se presenta denuncias

4. Supervisión punitiva por desconocimientos de los procesos de dirección y supervisión (gerenciales).

5. Ausencia de asesoramiento en los procesos técnicos administrativos y técnicos pedagógicos.

6. La gerencia del supervisor es por media tecnológica es decir por mensaje de texto o por mensajes de WhatsApp.

7. Ausencia de liderazgo y experiencia

8. Se da solo una supervisión de inspección y no de acompañamiento

9. Desconocimientos de funciones

10. La gerencia en Venezuela no existe, no existe jefe, solo acompañamiento pedagógico.

Fuente: Entrevista de los sujetos

Cuadro 2. Fortalezas del sistema de apoyo gerencial para la sustentabilidad de la supervisión educativa

\section{FORTALEZAS}

1. Recurso humano.

2. Planta física de la escuela.

3. Consejo Educativo.

4. Consejos Comunales.

5. Cocina y comedores.

6. Teorías.

7. Diferentes leyes que le dan sustento jurídico a la supervisión educativa.

Fuente: Entrevista de los sujetos

\section{Indagar sobre las políticas públicas que dan sustento a la supervisión educativa.}

Este objetivo responde a todas las categorías: carencia de formación del supervisor o la supervisora, carencia de las prácticas pedagógicas y administrativas. En este sentido, en los Cuadros 3 y 4 se observan las debilidades y las fortalezas, respectivamente. 
Cuadro 3. Debilidades de las políticas públicas que dan sustento a la supervisión educativa

\section{DEBILIDADES}

1. Desconocimientos de las políticas educativas.

2. Distorsión de los lineamientos en los circuitos educativos.

3. Debilidad en la aplicación de los lineamientos, circulares, normas.

4. Necesidad de formación para evitar que el proceso sea punitivo, no hay concordancia con lo que exige en el territorio y el accionar de las prácticas pedagógicas.

5. Desconocimiento de las normas y leyes e interpretación de las mismas.

6. Desconocimientos en el ordenamiento jurídicos, normas y reglas Abandono de parte del docente al Programa de Formación.

7. Ausencia de instrumento que el supervisor debe aplicar y le permita evaluar, valorar y medir los procesos.

8. Necesidad que surja una universidad con un pensum de estudio para la formación del supervisor, y no responda bajo a la práctica del amiguismo sino bajo a la práctica de la necesidad del territorio, sin sacar un docente de aula sin conocimiento que violentan las normas.

9. Debilidad en el currículo, la trasformación debe darse allí.

10. Politización del sistema educativo.

11. Ausencia de formación del personal en todas las especialidades.

Fuente: Entrevista de los sujetos

Cuadro 4. Fortalezas de las políticas públicas que dan sustento a la supervisión educativa

\section{FORTALEZAS}

1. Constitución República Bolivariana de Venezuela

2. Plan de formación docente PNF Y PNFA,

3. Convención colectiva

4. Arte 43 de la LOE

5. Circular 003013

6. Orientaciones pedagógicas

7. Programa Alimentación Escolar

8. Dotación de Canaima, Colección bicentenario, bultos escolares

Fuente: Entrevista de los sujetos

- Develar las funciones de los supervisores educativos para el alcance de las políticas públicas y las prácticas educativas

Este objetivo respondes todas las categorías: supervisión punitiva, y carencia de las prácticas pedagógicas y 
administrativas. En este sentido, en las Cuadro 5 se observan las debilidades.

Cuadro 5. Debilidades de las funciones de los supervisores educativos para el alcance de las políticas públicas y las prácticas educativas

\section{DEBILIDADES}

1. Divergencia de la teoría en práctica por parte de la supervisión.

2. Debilidad en formación académica, en relación a que todo el personal designado como supervisor debe estar formado en todas las disciplinas.

3. Ausencia de personal con funciones de supervisor, renuncias, abandono.

4. Desconocimiento de funciones.

5. No hay concordancia con lo que exige en el territorio y el accionar en las prácticas pedagógicas.

6. Necesidad de un sistema que dé respuesta a las insuficiencias de la supervisión

7. La gerencia es desplazada por el acompañamiento pedagógico, no existe gerencia.

8. Ausencia de competencias en los profesionales de la supervisión para el desarrollo de sus funciones.

9. Debilidad en el programa de Formación Avanzada de Supervisión y Dirección

10. Necesidad de experiencia en el territorio.

11. Ausencia de una estructura de apoyo gerencial, no existe un sistema nacional de supervisión.

12. Ausencia de experiencia.

13. Debilidades en el dominio de contenidos y desconocimiento sobre el significado de los ejes integradores y de estrategias para los proyectos de aprendizaje.

Fuente: Entrevista de los sujetos

\section{CONCLUSIONES}

$\mathrm{D}$ e acuerdo con el objetivo general de la investigación, el cual fue teorizar acerca de los sistemas de apoyo gerencial para la sustentabilidad de la supervisión educativa y su incidencia en las políticas públicas, el análisis y discusión de los resultados arrojó como conclusiones las siguientes:

Se determinó que no existe un sistema de supervisión nacional, no existe una estructura de apoyo gerencial, no hay una evidencia clara acerca de la concepción de la supervisión ni criterio alguno 
para planificar la acción supervisora; se conduce es a la transformación en la práctica con el acompañamiento pedagógico, enfocándose que la gerencia educativa que es desplazada por el acompañamiento pedagógico, como lo expresa el art. 43 de Ley Orgánica de Educación [LOE] (2009), no existe en Venezuela, lo que conlleva la debilidad de la aplicación de los procesos gerenciales. Por lo que no se evidencia el manejo, por parte del supervisor, de los aspectos técnicos administrativos, técnicos pedagógicos, por presentarse la ausencia de formación, de capacitación, de experiencia del personal supervisor designado en el territorio.

Existe una destacada concordancia entre los informantes sobre el desconocimiento de los supervisores del ordenamiento jurídico enmarcado desde la constitución nacional, la LOE, los reglamentos vigentes, circulares, contrataciones colectivas, entre otras; aunado a ello las deficiencias de los niveles de comunicación entre las instancias centrales, zonales, municipales, circuitales con las organizaciones educativas del territorio.

Se evidenció la ausencia, seguimiento y control del proceso educativo, según el trabajo del supervisor en el territorio y la respectiva evaluación de desempeño de los docentes con función supervisora.

Los sujetos encuestados coincidieron al manifestar que existe un plan nacional de formación avanzada de dirección y supervisión, impartido por la micro misión Simón Rodríguez con debilidades, lo que ha provocada la deserción de los docentes que se encontraban inscritos en este y su desplazamiento hacia la Universidad Pedagógica Experimental Libertador (UPEL) y la Universidad Nacional Abierta (UNA).

Se evidencia una gran divergencia entre las escuelas adscritas al Ministerio del Poder Popular para la Educación y las adscritas a la Secretaria de Educación del Gobierno del Distrito Capital, en cuanto al surgimiento de los circuitos educativos.

Existe una falta de transparencia en el proceso de selección del docente con función supervisora, persisten las prácticas viciadas y clientelares en detrimento de la valoración de la formación, de la experiencia académica, de la ética y el compromiso de la transformación educativa. 


\section{REFERENCIAS}

Bonilla, L. (2014). Calidad de la Educación y etapas de la Transformación educativa en Venezuela. Recuperado de https://www.aporrea.org/educacion/a186036.html

Constitución de la República Bolivariana de Venezuela (1999). Gaceta Oficial No. 36.860, de fecha 30 de diciembre de 1999

Denzin, N. y Lincoln, Y. (2005). The Sage Handbook of Qualitative Research. Thousand Oaks: Sage Publications The Discipline and Practice of Qualitative Research , 1/13

Husserl, E. (1962). Problemas fundamentales de la fenomenología. Madrid: Alianza

Ley Orgánica de Educación (2009). Gaceta Oficial № 5.929 extraordinario, 15 de agosto de 2009

Maldonado, C. (2008). Evaluación de Desempeño Docente en el Proceso de Enseñanza- Aprendizaje de los Estudios Básicos Supervisados del Instituto Universitario Tecnológico de Maracaibo (Trabajo de grado). Universidad Nacional Abierta, Caracas, Venezuela

Martínez, M. (2004). Ciencia y Arte en la Metodología Cualitativa. México: Trillas

Mogollón A. (2006). Funciones de la Supervisor Escolar en Venezuela, Aproximación a un Modelo (trabajo de grado). Universidad de Costa Rica. Facultad de Educación. Instituto de Investigación en Educación

Néreci, I. (1975). Introducción a la supervisión escolar. Argentina: Kapelusz. S.A

Ocando, C., H. Y. (2017). La supervisión educativa como elemento clave para alcanzar la calidad educativa en las escuelas públicas Omnia, 23(3), septiembre-diciembre, pp. 42-57. Recuperado de https://www.redalyc.org/pdf/737/73754947004.pdf

Palella, S., y Martins, F. (2004). Metodología de la investigación cuantitativa. Caracas: FEDUPEL

Pérez, R. (1998). ¿Existe el método científico? Historia y realidad. México: Fondo de Cultura Económica

Rojas, B. (2010). Investigación cualitativa. Caracas: FEDUPEL

Ruiz, N. (2005). Aproximación a un Modelo teórico de supervisión escolar para propiciar la excelencia de la Educación (tesis doctoral). Universidad Santa María, Caracas, Venezuela

Schütz, A. (1974). El problema de la realidad social. Buenos Aires: Amorrortu

Strauss, A. y Corbin, J. (2002). Bases de la Investigación Cualitativa. Colombia: Universidad de Antioquia 
Taylor, S. y Bogdan, R. (1987). Introducción a los métodos cualitativos de Investigación. Barcelona: Ediciones Paidós Ibérica, S.A

Vásquez T., D. Z. (2016). Ontopercepción de la supervisión educativa en el marco de la transformación social (trabajo de grado). Universidad de Carabobo, Valencia, Venezuela. Recuperado de http://mriuc.bc.uc.edu.ve/bitstream/handle/123456789/3367/dvasq uez.pdf?sequence $=1$ 